\title{
Lidil
}

Revue de linguistique et de didactique des langues

43 | 2011

Le rapport au savoir dans les discours professionnels

\section{La stagiaire sage-femme devant sa cliente : un double rapport au savoir}

The intern midwife and her client: a twofold relationship to knowledge

\section{Marty Laforest}

\section{(2) OpenEdition}

\section{Journals}

Édition électronique

URL : http://journals.openedition.org/lidil/3103

DOI : 10.4000/lidil.3103

ISSN : 1960-6052

Éditeur

UGA Éditions/Université Grenoble Alpes

\section{Édition imprimée}

Date de publication : 30 mai 2011

Pagination : 27-40

ISBN : 978-2-84310-201-1

ISSN : $1146-6480$

\section{Référence électronique}

Marty Laforest, «La stagiaire sage-femme devant sa cliente : un double rapport au savoir », Lidil [En ligne], 43 | 2011, mis en ligne le 30 novembre 2012, consulté le 19 avril 2019. URL : http:// journals.openedition.org/lidil/3103; DOI : 10.4000/lidil.3103

(c) Lidil 


\title{
La stagiaire sage-femme devant sa cliente : un double rapport au savoir
}

\author{
Marty Laforest*
}

\begin{abstract}
RÉSUMÉ
Un double statut d'étudiant et d'expert caractérise la situation du stagiaire. Posant l'hypothèse que la tension qui s'exerce entre ces deux statuts est repérable dans le discours, nous avons analysé, dans une perspective interactionniste, le discours de deux stagiaires sages-femmes québécoises lors de consultations de suivi prénatal. Un double rapport au savoir acquis et à transmettre apparait, qui est étroitement lié à l'histoire récente de la profession de sage-femme au Québec. Les sages-femmes québécoises fondent leur identité professionnelle sur un ensemble de valeurs distinctives, parmi lesquelles le partage du savoir médical avec la cliente occupe une grande place. L'analyse montre l'adhésion de la stagiaire à cette valeur; en cela, elle est déjà sage-femme. Mais le partage du savoir suppose une évaluation de la pertinence des informations à transmettre. Sur ce plan, la stagiaire reste une étudiante et doit encore acquérir un élément important de l'expertise : la souplesse dans le maniement de ce savoir.
\end{abstract}

\section{ABSTRACT}

A dual status as student and expert characterizes the situation of the student midwife. Hypothesizing that the tension between the two statuses is discernable in discourse, we analyzed, from an interactionist perspective, the discourse of two Québec student midwives during prenatal consultations. A twofold relation to the knowledge acquired and to be transmitted appears, which is closely tied to the recent history of the profession of midwifery in Québec. Québec midwives base their professional identity on a set of distinctive values, among which the sharing of medical knowledge with the client occupies a large place. The analysis shows that the students adhere to that value; in this, they are already midwives. But the knowledge sharing presupposes an evaluation of the relevance of the information to be transmitted. On this

* Département de lettres et communication sociale, Université du Québec à Trois-Rivières. 
level, the prospective midwives are still students and have yet to acquire an important element of their expertise: flexibility in handling medical knowledge.

Il ne sera pas question, ici, du discours du formateur, mais du discours de la personne que l'on forme, à ce moment clé qu'est le stage. Je tenterai de montrer comment la situation de stage oblige le stagiaire à gérer une tension s'exerçant entre sa position d'expert (devant l'usager de ses services) et d'étudiant (devant le superviseur de stage). Cette tension serait repérable dans l'interaction professionnelle, le discours du stagiaire articulant tant bien que mal un double rapport au savoir acquis (dans la formation) et à transmettre (à l'usager des services).

L'analyse, menée dans une perspective interactionniste, est fondée sur un corpus d'une vingtaine d'heures d'interactions sage-femme / cliente recueillies en 2003 à Montréal (données sur lesquelles je reviendrai un peu plus loin). Le savoir dont il est question est à la fois le savoir médical lié à la périnatalité et le savoir «de conviction» (pour reprendre l'heureuse expression de N. Blanc), relevant de la philosophie distinctive des sages-femmes québécoises.

\section{L'histoire singulière des sages-femmes québécoises}

La profession de sage-femme a connu au Québec une histoire singulière. Sans la rappeler en détail (voir Laforce, 1987; Vadeboncœur, 2004), mentionnons que les sages-femmes de village, présentes dans la tradition québécoise comme partout ailleurs, ont peu à peu disparu à mesure que l'hospitalisation des femmes devenait le standard en matière d'accouchement, à partir des années 1930, pour ne renaitre que dans les années 1970, en partie sous l'impulsion du mouvement féministe. La reconnaissance officielle de l'État en 1999, malgré les réticences du Collège des médecins, est l'aboutissement d'une longue lutte.

Les sages-femmes québécoises assument aujourd'hui la pleine responsabilité du suivi d'une grossesse normale, de l'accouchement et de la période postnatale. Les «nouvelles» sages-femmes se définissent par opposition aux autres groupes de professionnels œuvrant dans le domaine de l'obstétrique. Toujours pour l'instant très minoritaires dans le monde de la santé ${ }^{1}$, elles militent contre la technicité et la compar-

1. En 2006, seulement 1,6\% des naissances étaient supervisées par une sagefemme (0,5\% en 1994) [Ministère de la Santé et des Services sociaux, 2008, p. 25]. Les deux tiers des Québécoises font appel à un gynécologue. 
timentalisation croissantes des soins. Leur savoir technique et scientifique en matière de périnatalité est équivalent à celui d'un médecin généraliste. Elles s'en distinguent et se définissent donc par un certain nombre de valeurs spécifiques qu'elles défendent et qui orientent leur pratique, notamment une approche holistique de la santé et la volonté d'empowerment de la cliente (Ordre des sages-femmes du Québec, 2002), terme entendu comme le processus de soutien et de renforcement du pouvoir d'agir sur soi-même et sur son environnement (Le Bossé, 2003). Presque toutes les sages-femmes travaillent en maison de naissance, lieu d'accouchement et de consultation distinct de l'hôpital.

\section{La formation des sages-femmes}

$\mathrm{Au}$ Québec, la formation (universitaire) d'une sage-femme dure quatre ans. La première année est consacrée aux cours fondamentaux (anatomie, microbiologie et autres sciences biomédicales). À partir de la deuxième année, les étudiantes commencent une série de stages. Les cours sont dès lors donnés en ligne ou à un rythme intensif dans un bref laps de temps, car la formation se déroule en grande partie en maison de naissance. L'étudiante est alors couplée à une sage-femme expérimentée qui devient sa préceptrice. La stagiaire suit la préceptrice dans toutes ses activités, qu'il s'agisse des consultations, des accouchements ou des diverses réunions de travail et peu à peu, après une période d'observation, elle accomplit un nombre croissant de tâches. Chaque stage ne durant que quelques mois, l'étudiante a l'occasion de connaitre différentes préceptrices et manières de faire. C'est au cours de ces stages qu'elle acquiert la compétence technique et communicationnelle essentielle à la pratique et forge peu à peu son identité professionnelle.

\section{Données de l'analyse et méthodologie}

Je suis engagée dans la recherche sur et avec les sages-femmes depuis 2002. Cette démarche procède d'un cumul d'expériences d'observation participante et de collecte de données variées qui permettent de suivre l'évolution rapide de la profession. Les interactions sage-femme/ cliente $^{2}$ dont il sera ici question ont été enregistrées (enregistrement audio seulement) dans une maison de naissance, sans la présence d'un

2. Le terme cliente utilisé dans ce texte est celui que les sages-femmes ellesmêmes ont choisi d'utiliser après mure réflexion. Il n'est pas question pour 
enquêteur. Deux stagiaires et leurs préceptrices ont participé à l'étude. La première, Anne, effectue son deuxième stage; la seconde, Luce, achève son troisième stage. L'analyse présentée ici porte sur 9 des 16 consultations prénatales du corpus, soit celles où l'activité langagière de la stagiaire est importante, parce que la préceptrice lui confie plus ou moins brièvement la conduite de l'entretien. La stagiaire agit alors, en présence de sa préceptrice, en qualité de sage-femme devant la cliente. J'ai extrait de ces passages les séquences thématiques où la stagiaire se livre à l'explication ou au conseil, activités qui supposent l'étalage d'un certain savoir et qui de ce fait me semblaient susceptibles de présenter la trace d'un positionnement par rapport à ce savoir. 18 séquences ont été extraites des 9 consultations retenues (soit environ 20 pages de transcription). Faisant l'hypothèse qu'une certaine tension entre les statuts d'experte et d'étudiante se manifesterait dans le discours tenu par la stagiaire, j'ai concentré mon attention sur les passages où le savoir médical et les valeurs spécifiques à la profession (savoir de conviction) s'affichaient, afin de voir comment cette démonstration de savoir s'effectuait et ce qu'elle révélait.

\section{Le stage, un pivot dans la chaine de transmission du savoir}

L'analyse du discours de la stagiaire, dans les moments où elle dirige l'entretien, atteste qu'elle se trouve en position délicate, puisque si elle joue les professionnelles de la santé, elle a pleinement conscience de rester en même temps une étudiante dont toutes les actions sont susceptibles d'être évaluées. Ce statut hybride peut être schématisé de la façon suivante :

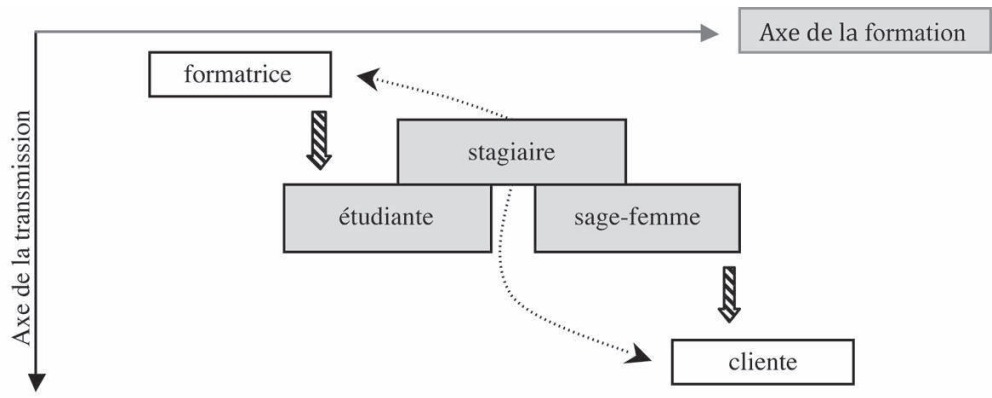

Figure 1. - Position de la stagiaire dans la chaine de transmission du savoir. 
L'axe de la formation représente le parcours (en gris) d'une candidate à la profession, de l'admission au programme à l'obtention du permis de pratique. Pour sa part, l'axe de la transmission représente la chaine sans fin des individus à qui est transmis un savoir qu'ils transmettent à leur tour. Le schéma n'en représente que trois «maillons», bref segment qui va de l'enseignante formatrice à son étudiante et de celle-ci (une fois diplômée) à la cliente. Les formatrices des futures sages-femmes qui enseignent à l'université ont exercé pour la plupart leur profession de sage-femme avant d'entreprendre les études supérieures qui leur ont permis de devenir professeures. Elles sont les dépositaires d'un savoir obstétrical produit par des scientifiques et d'un savoir expérientiel, d'un savoir-être intimement lié aux valeurs spécifiques à la profession. En tant que professeures, ce sont ces savoirs qu'elles transmettent, dans le cadre d'un programme universitaire, à leurs étudiantes. Celles-ci, une fois devenues sages-femmes, vont à leur tour contribuer à la transmission du savoir à la cliente. Bien sûr, à ce stade, la transmission change de nature, puisqu'il ne s'agit pas de faire de la consultante une experte, mais seulement de lui fournir, à la demande, l'information utile. C'est à cette étape de la transmission que se construisent à la fois l'autorité professionnelle de l'experte et la qualité de la relation avec la cliente. Car il s'agit à la fois de démontrer son savoir (c'est l'expertise) et d'en rendre une partie accessible (ce qui suppose le recours à diverses stratégies de vulgarisation et d'adaptation au profane). Le partage du savoir revêt une grande importance pour les sages-femmes et rejoint deux éléments essentiels de leur philosophie, soit l'établissement d'un rapport égalitaire avec la cliente et l'empowerment des futurs parents.

La stagiaire occupe une position singulière sur ces deux axes. Sur l'axe de la formation, le stage représente une étape charnière entre le statut d'étudiant et celui de professionnel. Sur l'axe de la transmission, la stagiaire occupe aussi une position intermédiaire : comme professionnelle, elle fait ses premières armes dans la transmission expert-profane du savoir, mais en même temps, en tant qu'étudiante qui agit en présence de sa préceptrice, elle doit faire la démonstration de l'acquisition du savoir qui lui a été transmis. Simultanément donc, dans l'interaction qui se construit avec la préceptrice et la cliente au cours de la consultation, elle rend compte de la qualité d'une transmission de savoir (ce qu'indique la ligne pointillée vers la formatrice dans la figure 1) et

elles d'appeler patiente la femme qui recourt à leurs services, la grossesse n'étant pas une maladie. 
transmet une partie de ce savoir (ce qu'indique la ligne pointillée vers la cliente).

La présentation schématique de la double position, et de ce fait de la double tâche discursive de la stagiaire sage-femme, permet de comprendre les divers cas de figure de ses interventions - qu'il s'agisse d'explications ou de conseils, dont je vais maintenant donner quelques exemples.

\section{La stagiaire en tant que sage-femme}

Les séquences d'explication ou de justification des conseils prodigués, assez fréquentes dans les données, font apparaitre un rapport au savoir nettement lié aux valeurs spécifiques à la profession et ce, à deux égards : premièrement, le savoir médical doit être largement et généreusement partagé; deuxièmement, les pratiques médicales doivent faire l'objet d'un examen critique ou du moins peuvent être mises en débat.

\section{Un savoir largement partagé}

Les données font apparaitre le savoir comme une chose qu'on ne garde pas pour soi, afin d'instaurer un rapport de pouvoir, conformément à l'optique des sages-femmes, suivant laquelle la concentration du pouvoir entre les mains des professionnels de la santé entraine la montée d'un sentiment croissant d'incapacité chez les femmes. On observe plusieurs traces d'établissement d'un rapport peu hiérarchisé, assez familier avec la cliente, ainsi que le recours quasi-systématique à l'explication des phénomènes dont il est question et à diverses stratégies de vulgarisation comme la métaphore ou la comparaison. L'exemple 1, dans lequel la stagiaire répond à une cliente qui craint de ne pouvoir faire face à un accouchement interminable, illustre bien cette façon de faire.

Ex. $1^{3}$ (extrait 5, MN2003, act. 10) :

3. Conventions de transcription: $\mathrm{STA}=$ stagiaire; $\mathrm{SF}=$ sage-femme; $\mathrm{CL}=$ cliente. La ponctuation est utilisée pour donner une idée de l'intonation. Lorsqu'aucun signe ou indication n'apparait, les mots s'enchainent sans aucune pause. Les mots soulignés indiquent un chevauchement de parole entre deux interlocuteurs. Les symboles suivants sont utilisés : «:» (allongement de la voyelle); «.» (intonème terminal); «,» (brève pause); «?», «! » (intonation clairement interrogative ou exclamative); « $(\mathrm{xxx}) »$, «(mots)» 
STA [l'accouchement] c'est un peu comme un hiking tu sais mais, une <ouais $>$ une excursion en montagne où tu escalades, <hum> tu sais, puis là tu montes, puis aaaff! tu sais c'est fatigant là un petit peu mais tu sais le début c'est, la phase de latence, puis là tout d'un coup tu commences à : dans les rochers un petit peu plus abrupts, pis tu travailles plus fort t'es dans la phase active, là t'arrives presque au sommet puis là t'as mal $: \leq$ (rire) $>$ t'as mal partout mais tu vois le but, pis là comment on s'encourage là, $<$ humhum $>$ tu sais dire «ok on arrive au bout on arrive au bout !» tu sais, <hum> on accepte cette douleur-là, <hum> parce qu'on sait, qu'on a quasiment fini $(\mathrm{xxx})$ c'est une belle : comparaison je trouve.

\section{Un savoir critiqué}

Les mentions, par la stagiaire, d'une source quelconque du savoir scientifique sont rares (on en trouve huit dans tout le corpus), peu variées et assez vagues : on informe la cliente sur tel sujet et à l'occasion, on mentionne «des études» ou «des recherches» menées sur ce sujet. Le fait intéressant est que presque toutes ces mentions surviennent dans le contexte d'une décision que les parents sont invités à prendre, elles relèvent donc de la mise en œuvre du choix éclairé, fondamental pour les sages-femmes. Elles ont par conséquent toujours une valeur argumentative et praxique très forte, d'autant plus que, dans plusieurs cas, il s'agit d'études qui n'ont pas été faites, information qui constitue un argument en faveur de la thèse que telle option comporte un risque parce que l'innocuité d'une pratique dont il est question n'a pas été démontrée. Il s'agit donc, dans ces cas qu'illustre bien l'exemple 2, de mettre en évidence, indirectement, le caractère distinctif des pratiques sages-femmes, en l'occurrence le choix laissé aux parents des décisions de soin et une grande prudence dans le recours aux médicaments de synthèse.

\section{Ex. 2 (extrait 11, MN2003, act. 13) :}

STA C'est à votre choix de, où est-ce que vous vous situez encore une fois avec les risques [de donner de la vitamine K au bébé]. [...] parce qu'il y a pas de, euh de recherches qui ont été faites à long terme, $<\mathrm{ok} \underline{\mathrm{xx}>\text { donc, }}$, sur le moment il y a peu de réactions, $[\ldots]$

(mots inaudibles ou dont la transcription est incertaine); « $[$ mots $] »$ (commentaire de l'analyste); «<mots>» (signal back-channel). 
mais à long terme il y a pas de recherches qui ont été faites, sur les, les conséquences que ça peut entrainer.

Tant en ce qui concerne le partage du savoir médical que sa critique, la comparaison du discours que tient la stagiaire avec celui que tient sa préceptrice à la cliente dans les mêmes circonstances ne fait pas apparaitre de traits distinctifs du discours de l'apprentie. Il appert que dans cette situation, la stagiaire, lors même qu'elle n'en est qu'à mi-parcours de sa formation, se comporte de manière très semblable à sa préceptrice (qu'elle a eu tout le loisir d'observer longuement), même si elle n'en a évidemment pas la finesse de jugement, l'aisance du geste et la facilité à interpréter le comportement de la cliente. Le rapport au savoir (partagé, critiqué) est celui qui est en accord avec la philosophie qui sous-tend la pratique et la stagiaire, en le reproduisant, montre que dès la deuxième année de formation, elle a commencé à faire siennes les valeurs spécifiques à la profession. La transmission du savoir de conviction et du savoir expérientiel des sages-femmes a donc bel et bien lieu ${ }^{4}$.

Ce rapport aux pratiques et au savoir obstétricaux est indissociable de l'histoire récente, très militante, de la profession. En effet, la renaissance des sages-femmes au Québec résulte d'une remise en question du pouvoir médical, perçu comme une mainmise sur le corps et la santé des femmes. Partager le savoir, c'est ne pas en faire un instrument de domination; le critiquer, c'est s'inscrire en faux contre la médicalisation à outrance d'un processus physiologique normal.

\section{La stagiaire en tant qu'étudiante}

Par ailleurs, le discours de la stagiaire montre qu'elle a pleinement conscience de rester une étudiante, bien que ce rôle soit moins en évidence que celui de professionnelle devant la cliente. Sur ce plan, l'influence de la sage-femme en titre se fait nettement sentir. Celle-ci, qui elle aussi joue deux rôles dans la consultation, donne comme la stagiaire priorité à celui qu'elle joue auprès de la cliente. Le rôle de préceptrice reste discret (il ne faut pas donner l'impression à la cliente qu'elle n'est là que pour faciliter l'apprentissage de l'étudiante), mais émerge par moments, ce qui recadre aussitôt la stagiaire dans le rôle

4. Ce qui confirme les résultats obtenus avec une tout autre méthode dans Laforest et Vincent (2007), où nous montrions que la première année de stage marque un point tournant dans l'intégration de ces valeurs par l'étudiante. 
d'étudiante. Il arrive ainsi parfois que la préceptrice pose une question à la stagiaire, mais toujours de manière assez fine, en l'invitant à fournir à sa place une information à la cliente. Celle-ci n'est donc jamais mise de côté, même dans ces moments où le rôle de préceptrice est brièvement dominant, comme le montre l'exemple 3.

\section{Ex. 3 (extrait 5, MN2003, act. 10) :}

1 SF des contractions entre 5 et 20 minutes, ça c'est la phase de latence, c'est latent

2 c'est préparatoire, ça appelle les invités au party [à la fête]

3 CL Ça peut durer, longtemps.

4 SF 10 à 12 heures [...]

5 mais c'est pour ça que l'erreur que les gens font c'est de sss c'est quoi?

6 CL Ben

7 STA Ben de : de rester debout de calculer les contractions, puis d'attendre la progression,

$8<$ CL. hum > puis pendant ce temps-là ils se reposent pas.

On notera dans ce passage la transition subtile d'une destinataire à l'autre : le début de l'intervention, dans lequel la sage-femme explique ce qu'est la phase de latence, s'adresse sans équivoque à la cliente. À la ligne 5, l'auto-interruption («c'est de sss c'est quoi ?») indique qu'en cours de formulation la sage-femme transforme en question ce qui devait être une affirmation. Cette question ne peut s'adresser qu'à la stagiaire. La cliente s'y trompe et tente un début de réaction (ligne 6), mais presque simultanément, la stagiaire répond à sa place (lignes 7-8). Ce faisant, elle se trouve à poursuivre ce qui apparait (quand on considère l'ensemble de la séquence dont l'exemple est tiré) comme une seule intervention complexe visant à calmer l'inquiétude de la cliente à propos de la durée de l'accouchement, intervention co-construite par la préceptrice et la stagiaire. Un seul point de vue, celui de «l'expertise», s'exprime, la co-énonciation préservant la prééminence de l'interaction sage-femme/cliente sur l'interaction préceptrice/stagiaire.

Dans tous ces cas, il y a référence indirecte au maillon antérieur de la chaine de transmission du savoir, c'est-à-dire au moment où le savoir passe de la formatrice à l'étudiante. C'est l'action de la préceptrice qui remet la stagiaire dans son rôle d'étudiante. Mais ce recadrage ne va pas toujours sans conséquence pour la cliente. En effet, le savoir dont la cliente a besoin n'est pas celui dont doit disposer le professionnel. En lui demandant de produire une explication à l'intention de la cliente, la 
sage-femme peut amener la stagiaire à construire un discours qui relève d'une démonstration d'acquisition du savoir médical allant bien au-delà de l'information qui répondrait à ses besoins. Dans ces cas relativement fréquents, le discours émancipatoire que tient la stagiaire comporte un si grand nombre de détails techniques qu'on se demande si la cliente en est véritablement bénéficiaire; il s'adresse au moins autant à la préceptrice qu'à cette dernière. L'exemple 4 illustre ce phénomène.

Ex. 4 (extrait 14, MN2003, act. 17) :

La sage-femme vient de demander à la cliente si on lui a déjà parlé de la vitamine $K$.

1 SF (à [...] est-ce que tu as une explication pour la vitamine $\mathrm{K}$ [...] À toi la parole.

2 STA)

3 STA [...] la vitamine K c'est pour prévenir la maladie hémorragique, du nouveau-né, $<\mathrm{CL}$.

4 ok> puis ça c'est euh : c'est des hémorragies, entre autres, euh : au niveau du cerveau

5 que le bébé peut faire, partout, mais, euh ils se sont rendu compte c'est dans les années

65060 les femmes étaient souvent endormies au moment de la poussée, du bébé,

7 SF Exact.

8 STA $(\mathrm{xx})<$ CL Oui oui $>$ tout ça. On poussait sur le bébé on prenait les forceps, et puis là on

9 le sortait comme ça, pis ça endommageait les capillaires, ça endommageait la tête des

10 bébés, pis là ils ont vu que les bébés ils faisaient des hémorragies, ok, puis c'est là qu'ils

11 ont commencé, à donner l'injection de la vitamine K, ok, mais : pour un bébé qui nait,

12 normal, avec une sortie, sans instrument euh : pas de traumatismes là crâniens tout ça,

13 nous ce qu'on suggère aux parents, ok, c'est euh : au moment de la naissance on voit la

14 naissance comment la naissance s'est passée, pis si le bébé a eu une naissance :

15 instrumentale avec une ventouse avec les forceps, euh si le bébé, a tombé est échappé

16 frappé peu importe tu sais (ça peut être) une (autre) indication, si le bébé reçoit plus de 
1720 minutes, d'oxygène, <CL Humhum> euh l'oxygène dilue un, des éléments, de

18 coagulation dans le sang il y en a douze, au moins, éléments de coagulation il en dilue

19 un, bon, s'il a reçu plus que 20 minutes d'oxygène on se dit «bien ça pourrait être, une

20 bonne indication prévention», euh si le bébé est prématuré parce que ses capillaires

21 sont beaucoup plus fragiles, euhm : si le bébé vient au monde avec un céphalhématome

22 ça ça vient d'un frottement os à os pendant la descente $\leq \mathrm{CL}$ ok> là c'est une

23 accumulation de sang, sous l'os, $<$ CL ok $>$ au niveau des sutures, ok, euh ça ça peut être

24 une autre indication tu sais il y a vraiment $\leq$ CL Ouais ouais $>$ des choses, précises, qui

25 nous disent, «ah ce petit bébé-là on lui en donnerait », tu sais, mais un bébé qui nait là,

26 sans rien là de, de tout ça là en bonne santé, où on n'a pas d'indications en résumé, mais

27 encore là tu peux vérifier les études avec ton conjoint pis, dire «bien on aimerait peut-

28 être le donner par précaution. Ou, on le veut pas. Ou, on va voir selon l'accouchement

29 là », pis euhm la dose, qui est donnée, au bébé, est, je vais dire 10000 fois là mais : le

30 chiffre que j'ai vraiment en tête c'est 100000 c'est ça il faudrait que j'aille le vérifier

31 parce que je suis toujours en train de boguer sur ce chiffre-là, la dose, que le bébé a

32 besoin, ok, <CL ( $\mathrm{xx})>$ c'est énorme, comme quantité, euh : le bébé en a pas, de

33 vitamine $\mathrm{K}$ dans son système à la naissance, parce que la vitamine $\mathrm{K}$ elle est faite avec

34 euh : dans l'intestin, ok? <CL ok> par la digestion avec des enzymes pis tout ça puis là

35 lui il arrive avec l'intestin stérile il a pas encore toutes ses enzymes puis tout ça, mais il

36 en reçoit, de la maman, un petit peu, dans le lait maternel pis lui il va commencer à la

37 fabriquer, entre 5 et 20 jours à peu près là tu sais il commence à en fabriquer lui-même. 
$39 \mathrm{CL} \quad$ Non [2 sec.]

40 STA Ok. Fait que, vous regarderez ça ensemble [...]

$41 \mathrm{CL} \quad$ [très très bas] Oui

42 SF Oui euh : [2 sec.] c'est beau. Bonnes explications.

Ce qui frappe dans ce passage, c'est la profusion de détails techniques dont la pertinence apparait discutable pour la cliente. En effet, la tâche de la stagiaire consiste à mentionner qu'on proposera après la naissance de donner une dose de vitamine $\mathrm{K}$ au bébé, injection qui n'est vraiment nécessaire que dans le cas d'une naissance traumatique. Mais la stagiaire fait beaucoup plus : elle énumère notamment toutes les indications de cette injection (lignes 14-25), dont la mention est parfois accompagnée d'une explication (par exemple, le rôle de l'oxygène dans la coagulation du sang, ligne 17, ou la cause du céphalhématome, ligne 22), avant d'évoquer la dose nécessaire (ligne 29) et le processus de fabrication de la vitamine K par l'organisme (lignes 33-37) ${ }^{5}$.

Il serait hasardeux d'estimer précisément le degré de pertinence de telle ou telle information. On peut cependant faire remarquer qu'une intervention à peu près monologale d'une telle longueur (près de sept minutes et demie) est rare dans les interactions sage-femme/cliente, qui présentent un certain équilibre de la parole entre les interlocutrices. Cet équilibre est ici nettement rompu. Un autre indice que l'intervention s'adresse au moins autant à la préceptrice qu'à la cliente est que la première y réagit davantage que la seconde. La réaction minimale de la cliente (lignes 39 et 41 ) peut être interprétée comme une marque de quasi-désintérêt (que dire après un tel déluge de paroles ?); la préceptrice, pour sa part, évalue la qualité de l'explication à deux reprises (ligne 7 : «exact» et ligne 42 : «C'est beau. Bonnes explications»).

Ce discours fait apparaitre un autre rapport au savoir, celui d'un savoir pas encore intégré, trop scolaire pour les besoins de l'interaction professionnelle avec la cliente, d'un savoir dont l'acquisition doit encore être prouvée. Il témoigne d'une nette tension entre les deux rôles

5. L'analyse de l'importante composante argumentative de cette intervention ne peut être développée ici, faute d'espace. Le point de vue qui s'y déploie à travers l'information donnée est encore une fois celui d'une remise en question des pratiques médicales routinières, accompagnée d'une affirmation de l'identité professionnelle distincte des sages-femmes («nous» [les sagesfemmes] ce qu'on suggère, [...] c'est..., ligne 13). 
d'étudiante et de professionnelle qu'endosse la stagiaire. Cette tension ne va pas sans conséquences interactionnelles, comme en témoigne l'apathie de la cliente à la fin de l'exemple, attitude rare dans les consultations recueillies.

Toutefois, on ne saurait passer sous silence le rôle que joue aussi peut-être, dans cet étalage de savoir médical, le statut toujours institutionnellement précaire des sages-femmes au Québec. Dix ans après sa reconnaissance officielle, la profession reste méconnue et victime de la représentation dominante dans la population, celle de la sage-femme d'antan, dépositaire d'un savoir populaire associé à la non-modernité et surtout à la non-sécurité (Vincent, Laforest et Deshaies, 2002), une image qu'a réussi à imposer l'institution médicale responsable de sa disparition. Dans ce contexte, la démonstration superfétatoire de savoir médical n'est pas seulement l'apanage d'une stagiaire qui veut bien faire, elle manifeste également l'intériorisation de la représentation qu'on se fait généralement au Québec de la sage-femme. Sur ce plan, la compétence à prouver ne caractériserait pas que l'attitude de la stagiaire, mais relèverait plus largement de l'ethos de celle qui exerce une profession en voie de légitimation.

\section{Conclusion}

Notre rapide analyse du discours de deux stagiaires sages-femmes permet de conclure que la stagiaire, dès la deuxième année de sa formation, a bien intégré plusieurs des valeurs de sa future profession et, notamment, fait du partage de son savoir - sans lequel une relation égalitaire avec la femme enceinte ne saurait s'établir - un impératif. Toutefois, l'hybridité de son statut, ni tout à fait celui d'une étudiante, ni tout à fait celui d'une sage-femme en titre, transparait dans son discours. En effet, s'il revient à la sage-femme de se faire la continuatrice de la chaine de transmission du savoir en émancipant la cliente autant que faire se peut, le savoir à transmettre change dans l'interaction professionnelle : la sage-femme n'est pas enseignante, mais conseillère et à cet égard, son rôle consiste non seulement à vulgariser son savoir médical de façon à le rendre accessible au profane, mais aussi à évaluer la quantité et la nature des informations qu'elle doit donner. Sur le plan interactionnel, on sait depuis longtemps que la rétention du savoir par le soignant peut avoir des effets néfastes sur la relation avec le soigné et sur la qualité du traitement (Fainzang, 2006; Richard et Lussier, 2007); mais une quantité trop importante d'informations peut également nuire en provoquant 
le désintérêt du soigné. Sur ce plan, le discours de la stagiaire qui, dans les passages analysés, tente de concilier ses deux rôles d'étudiante et de professionnelle, montre le caractère paradoxal de sa situation, mais aussi le savoir-faire qu'il lui reste à acquérir pour devenir une véritable sage-femme, en l'occurrence cette souplesse dans le maniement du savoir qui caractérise le professionnel aguerri.

\section{RÉFÉRENCES BIBLIOGRAPHIQUES}

FAINZANG S. (2006) : La relation médecins-malades. Information et mensonge, Paris, PUF.

LAFORCE H. (1987) : «Les grandes étapes de l'élimination des sages-femmes au Québec du $17^{\mathrm{e}}$ au $20^{\mathrm{e}}$ siècles », dans F. Saillant et M. O'Neill (dir.), Accoucher autrement. Repères historiques, sociaux et culturels de la grossesse et de l'accouchement au Québec, Montréal, Éd. St-Martin, p. 163-180.

LAFOREST M. et VincENT D. (2007) : «The transmission of values specific to midwifery ans their integration by students midwives », Canadian Journal of Midwifery Research and Practice, vol. 6, n 2, p. 18-26.

Le Bossé Y. (2003) : «De 1'“habilitation" au "pouvoir d'agir" : vers une appréhension plus circonscrite de la notion d'empowerment» [en ligne], Nouvelles pratiques sociales, vol. $16, \mathrm{n}^{\circ} 2, \mathrm{p} .30-51$. Disponible sur $<$ http://www.erudit.org/revue/nps/2003/v16/n2/009841ar.html> [consulté le 23 juin 2010].

Ministère de la Santé et des Services sociaux du Québec (2008) : Politique de périnatalité 2008-2018, Québec, Direction des communications du MSSS, $62 \mathrm{p}$.

OrdRe des SAGES-Femmes du QuéBec (2002) : «Philosophie» [en ligne], disponible sur $<$ http://www.osfq.org/philosophie.php $>$ [consulté le 23 juin 2010].

RichaRd C. et Lussier M.-T. (2007) : «Pourquoi s'intéresser à la communication médecin-patient à propos de la médication?», dans J. J. Lévy et C. Garnier (dir.), La chaine du médicament. Perspectives pluridisciplinaires, Québec, PUQ, p. 333-366.

VADEBONCEUR H. (2004) : «Delaying legislation: The Quebec experiment», dans I. L. Bourgeault, C. Benoît et R. Davis-Floyd (dir.), Reconceiving Midwifery, Montréal et Kingston, McGill-Queen's University Press, p. 91-110.

Vincent D., Laforest M. et Deshaies D. (2002) : La perception de la pratique sage-femme, Rapport inédit, Trois-Rivières, UQTR, 16 p. 